\title{
Uma Abordagem Computacional em Ambientes Assistidos Baseada em Atividades Físicas e Biometeorologia
}

\author{
João Pedro de Souza Jardim da Costa ${ }^{1}$ Mario Antônio Ribeiro Dantas ${ }^{1}$ \\ José Maria Nazar David ${ }^{1}$
}

${ }^{1}$ Departamento de Ciências da Computação - Universidade Federal de Juiz de Fora (UFJF) Caixa Postal 20.010 - 36036-900 - Juiz de Fora - MG - Brazil

pedrocxmegmail.com, mario.dantas@ice.ufjf.br, jose.davideufjf.edu.br

\begin{abstract}
The importance of performing physical activities to preserve personal health is a common knowledge of all human society. A question to be considered would be: is performance, when carrying out these activities, affected by the ecosystem that surrounds people? Affecting performance means affecting productivity and, on the other hand, it implies affecting the efficiency in carrying out the activity.This paper had as a proposal to present an application that aims to support professionals in the field of physical education in the accompaniment of their students, while taking into consideration the meteorological elements that affect them.Another differential contribution was the simulation developed aiming at a better visualization of the environment.
\end{abstract}

Resumo. A importância de realizar atividades físicas para preservar a saúde pessoal é um conhecimento comum a toda sociedade humana. Uma pergunta a ser considerada seria: o desempenho, ao realizar essas atividades, é afetado pelo ecossistema que cerca as pessoas? Afetar o desempenho significa afetar o rendimento e, em contrapartida, implica afetar a eficiência na realização da atividade.Este trabalho de pesquisa apresenta uma proposta de uma aplicação que visa amparar profissionais da área de educação física no acompanhamento de seus alunos, enquanto leva em consideração os elementos meteorológicos que os afetam. Outra contribuição diferencial foi a simulação desenvolvida visando uma melhor visualização do ambiente.

\section{Introdução}

O trabalho apresentado em Vihma (2010) ilustra estudos que as variáveis meteorológicas afetam o desempenho físico dos praticantes. Em Wachowicz, Małysiak-Mrozek e Mrozek (2019), também, existe um esforço na demonstração que elementos do clima influenciam as atividades físicas. O objetivo dessa pesquisa foi apresentar os resultados adquiridos em suas investigações sobre os impactos que pressão e umidade do ar, velocidade do vento, temperatura, índice UV, ponto de orvalho e formação das nuvens têm sobre corridas/caminhadas ao ar livre. Seus resultados experimentais indicaram que os atletas realmente são impactados pelo clima. Todavia, um aspecto interessante é que a intensidade do impacto é heterogênea, ou seja, existe uma variabilidade entre os atletas. Outro desafio foi considerar como capturar os dados digitais de saúde, como duração de um treino, a 
distância percorrida, os batimentos cardíacos e velocidade média. Após estudar o trabalho de Nascimento et al. (2020), escolhemos nos basear na utilização de smartwatches.

Nesse trabalho apresentamos um esforço no sentido de desenvolvimento de uma abordagem, através de um aplicativo, considerando o relacionamento das variáveis meteorológicas com o desempenho de seus usuários. Em adição, utilizando o simulador de contexto Siafu (2020), disponível em seu sítio ${ }^{1}$, construímos cenários para alimentar a aplicação e, assim, realizar testes com seus resultados.

Este documento foi organizado em quatro seções. Os materiais e métodos relativos à pesquisa são apresentados na seção 2 . Na seção 3 são resultados relativos à nossa proposta. As considerações finais são apresentadas na seção 4.

\section{Materiais e Métodos}

Nesta seção apresentamos o ambiente, as ferramentas, os métodos utilizados para produzir dados, gerar informações e observar os resultados.

\subsection{Cenário Simulado}

No mapa da simulação, baseado no bairro Graminha, da cidade de Santos Dumont, estado de Minas Gerais, existem dois tipos de locais, residência e trabalho. Definiremos a pessoa dentro da simulação como agente. Toda movimentação do agente é utilizada para calcular o seu desempenho físico, de acordo com o clima, sendo sua velocidade o valor medido. Além dos agentes, o cenário também simula elementos meteorológicos. Por motivos de limitação de hardware, apenas três elementos foram simulados: densidade do ar (Density of Air), temperatura do ambiente (Temperature) e umidade do ar (Humidity), às 00:00h, de cada dia, o valor dessas variáveis são mudados aleatoriamente. A densidade do ar, a temperatura e a umidade foram escolhidos pois, nos trabalhos relacionados a área, como

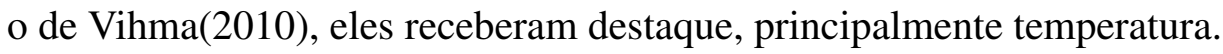

Definiu-se duas configurações do mesmo cenário com uma pequena, mas importante, diferença entre elas. Na primeira configuração, as variáveis meteorológicas tiveram o mesmo valor em todo o mapa, ou seja, se a temperatura teve o valor low em um ponto do mapa, então a temperatura teve esse mesmo valor em qualquer outro ponto do mapa. Valor considerado até ser mudado no fim do dia, não significando que temperatura, umidade e densidade do ar tiveram necessariamente o mesmo valor. Na segunda configuração fez-se o contrário: a variável meteorológica teve um valor geral e, também, variações em vários pontos do mapa; por exemplo, o valor da temperatura geral é low, mas em outras partes do mapa a temperatura tem o valor very low ou comfortable. É importante apontar que, na segunda configuração, foi considerado como valor geral o valor predominante no mapa. Dito isso, na simulação, sensores captavam o valor de acordo com o local em que o agente estava, enquanto o sistema meteorológico captava apenas o valor geral. Foram realizadas quatro simulações por configuração, cada simulação teve duração variável entre 60 e 64 dias.

\subsection{Solução Proposta}

A aplicação desenvolvida para este projeto de pesquisa teve como objetivo auxiliar profissionais da área de educação física no monitoramento do desempenho de seus alunos,

\footnotetext{
${ }^{1}$ http://siafusimulator.org/download
} 
levando em consideração a influência do clima. Na Figura 1 é ilustrado o cenário de uso idealizado.

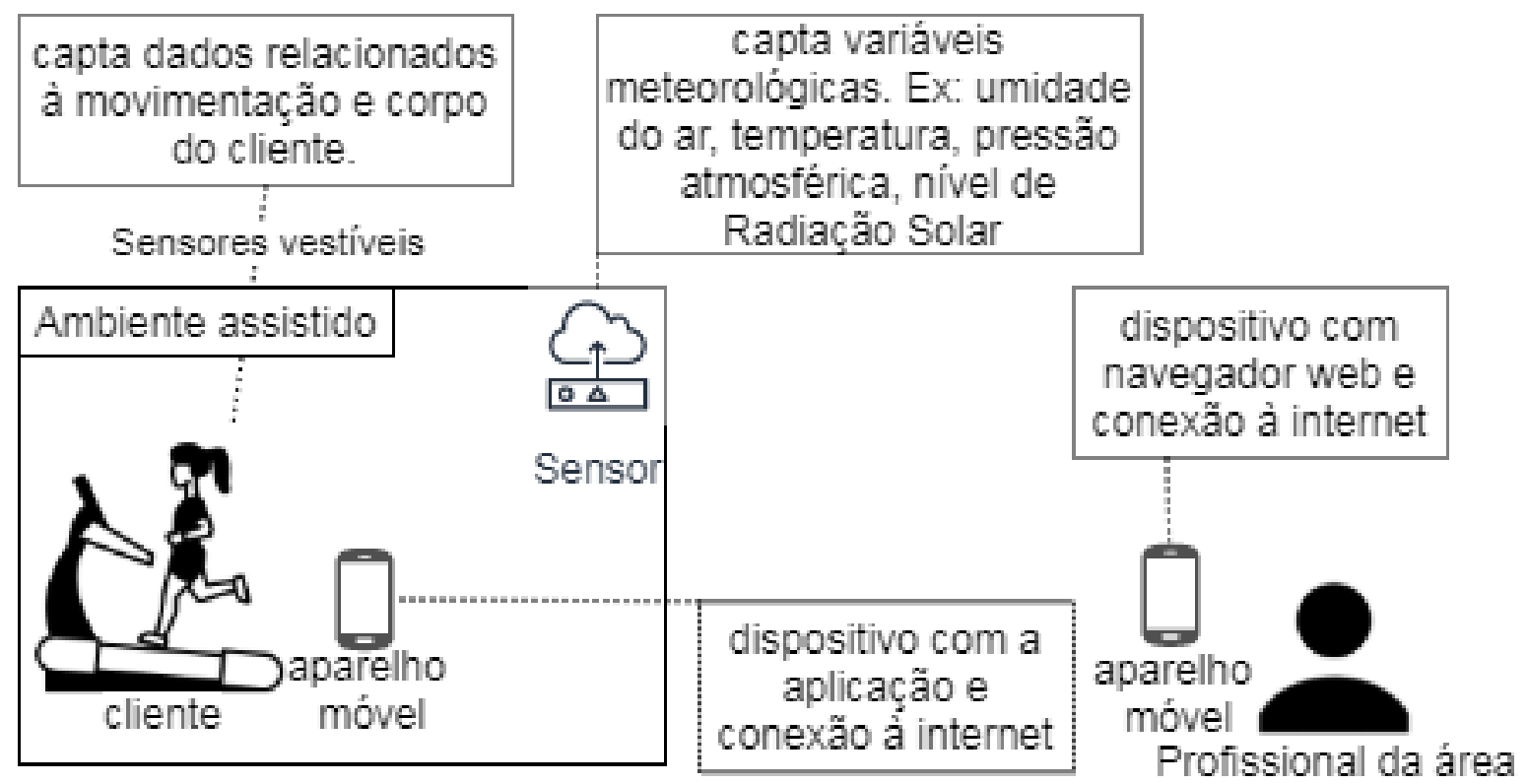

Figura 1. Modelo de alto nível que ilustra o cenário de uso da aplicação.

Dois cenários de uso para esta aplicação foram idealizados: no primeiro caso, ilustrado pela Figura 2, há o aluno utilizando sensores vestíveis, realizando atividades físicas em um ambiente equipado com sensores capazes de captar dados meteorológicos; paralelamente, o profissional responsável pelo aluno acessa a aplicação através de navegador web para acompanhar o desenvolvimento de seu aluno.

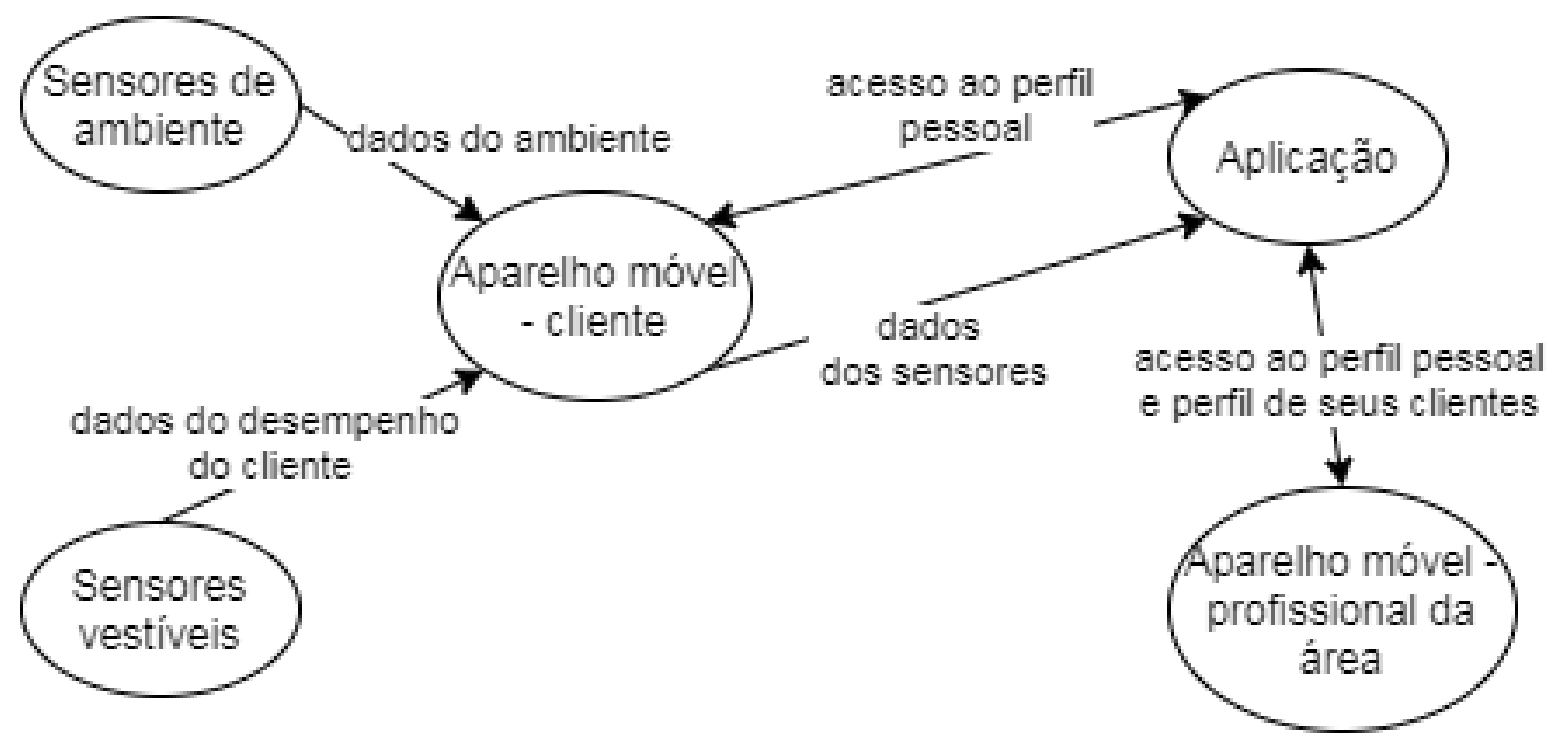

Figura 2. Diagrama de fluxo do primeiro cenário, onde são utilizados sensores para captar dados das variáveis meteorológicas.

No segundo cenário, a única diferença seria o fato de o aluno se encontrar em 
um local sem sensores que possam auxiliar na captação de dados das variáveis meteorológicas; nesse caso, a aplicação faria uso de dados sobre o clima local fornecidos por um sistema meteorológico. Na Figura 3 é ilustrado como o segundo cenário seria.

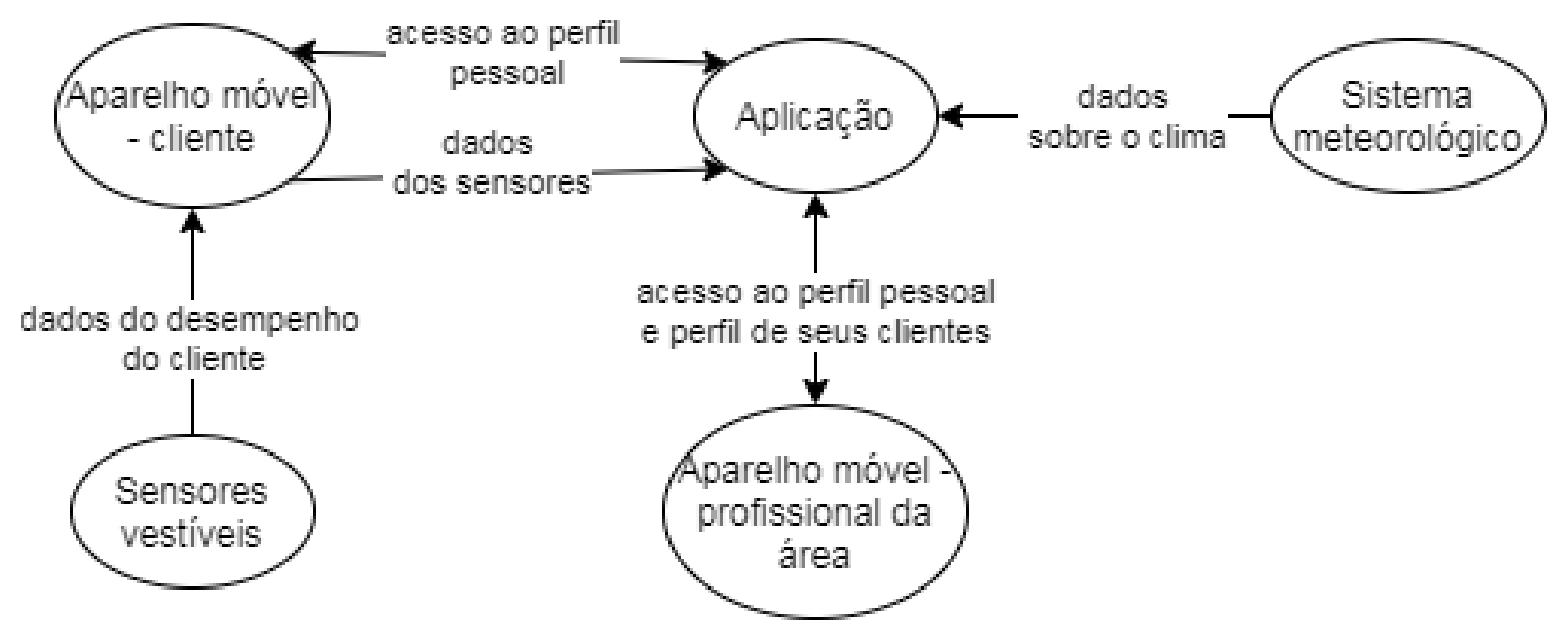

Figura 3. Diagrama de fluxo do cenário alternativo; neste, a aplicação é alimentada por dados fornecidos por um sistema meteorológico.

Ambos os cenários foram executados dentro do simulador Siafu (2021). Na simulação, foram considerados os dados de uso de sensores e os dos sistemas meteorológicos.

A informação mais importante para este documento é a estimativa de melhor e pior clima para o aluno. Na Figura 4 demonstramos como a aplicação apresenta essas informações.

Melhor e Pior Performance

\begin{tabular}{llll}
$\#$ & Temperatura & Umidade & Densidade do Ar \\
\hline Melhor Performance & low & high & low \\
\hline Pior Performance & high & low & high
\end{tabular}

Figura 4. Tabela com a estimativa, feita pela aplicação, da melhor e pior combinação de valores das variáveis meteorológicas para o desempenho do aluno.

A aplicação utiliza os dados, sobre o desempenho do aluno e o clima que o afetava, para estimar quais combinações de valores, das três variáveis meteorológicas, representariam os dois extremos para a performance do aluno, pior e melhor.

\subsection{Método de Teste}

Antes de abordar o método de teste, precisamos explicar as características do agente dentro da simulação. Cada agente possui um valor para cada variável meteorológica. Os va- 
lores possíveis são: strong low, weak low, strong comfortable, weak comfortable, strong high e weak high. Esses valores dizem respeito a como o corpo do agente responde àquele clima. Como exemplo, se a temperatura atual for low e o agente possuir o valor weak low para temperatura, sua velocidade de movimentação é diminuída, enquanto strong low teria o impacto contrário, sua velocidade aumentaria. Desta forma, com a combinação dos três valores que o agente possui e o clima do momento, é calculado a velocidade do agente. Referenciaremos a esses valores como características climáticas.

Como demonstramos na Figura 4, a aplicação estima dois valores para cada variável meteorológica, uma para pior e uma para melhor performance. Em nossos testes, associamos as estimativas de pior com o prefixo weak e as de melhor com o prefixo strong. Então, se na aplicação foi estimado que com temperatura high o agente terá sua melhor performance, verificamos se na simulação o agente possuía o valor strong high para temperatura. Assim, contamos quantas, das características climáticas, foram estimadas corretamente por cada agente.

\section{Resultados}

Utilizando o cenário como critério de separação por linha e a quantidades de estimativas corretas como critério de separação por coluna, na Tabela 1 são apresentados os resultados dos testes da função de estimativa da aplicação.

Tabela 1. Tabela com as porcentagens das estimativas. Cada coluna organiza os agentes em quantas de suas características climáticas foram estimadas corretamente, cada linha separa os em qual cenário faziam parte.

\begin{tabular}{|l|l|l|l|l|}
\hline Cenário & 0 acertos & 1 acertos & 2 acertos & 3 acertos \\
\hline $\begin{array}{l}\text { Sistema meteorológico } \\
\text { config. 2 }\end{array}$ & $40,00 \%$ & $40,63 \%$ & $17,50 \%$ & $1,87 \%$ \\
\hline Sensores config. 2 & $19,37 \%$ & $42,50 \%$ & $26,88 \%$ & $11,25 \%$ \\
\hline $\begin{array}{l}\text { Sistema meteorológico } \\
\text { config. 1 }\end{array}$ & $13,21 \%$ & $57,50 \%$ & $21,25 \%$ & $8,04 \%$ \\
\hline
\end{tabular}

A função de estimativa de melhor e pior performance demonstrou um padrão com simulações de até 64 dias, onde o maior grupo é de agentes que teve pelo menos 1 de suas características climáticas estimada corretamente, seguido pelo grupo que teve 2 estimadas corretamente. É possível observar esse acontecimento nas linhas 2 e 3 da Tabela 1; no entanto, o mesmo não acontece na linha 1. Para dar justificativa a essa igualdade entre os resultados das duas últimos linhas e diferença com a primeira, deve-se falar sobre as diferenças e igualdades nos processos realizados para criar tais resultados.

Nada foi mudado na aplicação; contudo, utilizou-se sistemas meteorológicos nas simulações das linhas 1 e 3, e sensores nas simulações da linha 2. Outra diferença está no clima; para as linhas 1 e 2 foi utilizado um clima misto, que possui valores diferentes ao redor do mapa, enquanto para a linha 3 foi utilizado um formato de clima ótimo para o sistema meteorológico, que tem o mesmo valor em todo o mapa, além do fato de serem dois conjuntos diferentes de simulações para os dois primeiros e para o último. Percebese que, em seu estado ótimo, a taxa de acerto com o sistema meteorológico se equipara à com sensores; mas, com o clima misto, há uma perda de dados que afeta negativamente o resultado final. 


\section{Considerações Finais}

Neste artigo propôs-se uma aplicação como ferramenta para apoiar profissionais da área de educação física no acompanhamento do desempenho de seus alunos, quando levado em consideração o clima do ambiente em que a atividade se desenvolverá. Outrossim, apresentou-se duas versões da aplicação; uma que capta dados do clima apenas por meio de sensores e outra que capta os dados através de sistemas meteorológicos. Por fim, estabeleceu-se uma comparação entre resultados obtidos nas duas versões.

Para criação da base de conhecimento, poucos documentos que abordavam a relação entre desempenho físico e o clima foram encontrados, e nenhum que abordava o lado de tecnologia da informação do problema foi encontrado. Desenvolver o protótipo de uma aplicação, sem base em precedentes, exigiu que fosse feito um estudo mais aprofundado. Para programar as ações dos agentes, foi necessário basear-se nos resultados dos testes em pessoas reais, realizados em documentos anteriores.

Utilizar o simulador de contexto Siafu provou ser um desafio; além de aprender sobre as funções de sua biblioteca, foi indispensável estudar maneiras de criar um cenário que solucionasse nossas necessidades. Um cenário simulado, mal construído, que não reflita o cenário real desejado, geraria dados inúteis à aplicação. Outro desafio foi a limitação de atividades que os agentes dentro da simulação podem realizar. Por causa da simplicidade de seus modelos, a única atividade realmente possível para os agentes é mover-se de um ponto do mapa a outro; isso, indiretamente, limitou a aplicação.

Sensores são a forma de captação de dados recomendada pois, além do fato de sistemas meteorológicos fornecerem valores gerais de uma região, os valores de certas variáveis meteorológicas podem ser artificialmente modificados quando dentro de locais fechados, com o uso de aparelhos. Utilizar a aplicação em tal situação traria resultados possivelmente imprecisos e diferentes do esperado, caso estivesse captando dados através de um sistema meteorológico.

\section{Referências}

Nascimento, M. G. d., Iorio, G., Thomé, T. G., Medeiros, A. A., Mendonça, F. M., Campos, F. A., David, J. M., Ströele, V., and Dantas, M. A. (2020). Covid-19: A digital transformation approach to a public primary healthcare environment. In 2020 IEEE Symposium on Computers and Communications (ISCC), pages 1-6. IEEE.

Siafu An Open Source Context Simulator (2007). Siafu home. Acesso em: 26, janeiro de 2020.

Vihma, T. (2010). Effects of weather on the performance of marathon runners. International journal of biometeorology, 54(3):297-306.

Wachowicz, A., Małysiak-Mrozek, B., and Mrozek, D. (2019). Combining data from fitness trackers with meteorological sensor measurements for enhanced monitoring of sports performance. In International Conference on Computational Science, pages 692-705. Springer. 